\title{
The stargardt disease experience: An analysis of expressive writing essays about living with a rare eye disease
}

\author{
Jennifer L Bryan*, Angie S LeRoy, and Qian Lu \\ University of Houston, Houston, USA
}

\begin{abstract}
Individuals with Stargardt disease, a rare eye disease, experience unique challenges with daily life. However, literature on psychological effects, potential areas of intervention, and gaps of care are sparse. This study explored these domains with a novel approach. Using grounded theory, we analyzed the expressive writing essays of 22 participants with Stargardt disease. Participants revealed frustration with the diagnosis process, loss of independence, social embarrassment, feeling misunderstood, and impact on their personal and professional lives. Positive experiences included effective coping strategies and social support networks. This study provides insight into the benefits of analyzing essays produced in expressive writing interventions among understudied populations. Results suggest communication between practitioners, patients, and their family is important especially during diagnosis. Future research and care providers could incorporate these findings in developing interventions to build patients' support systems and educate patient and families about the unique challenges of this rare disease.
\end{abstract}

\section{Introduction}

Stargardt disease (SD), a juvenile form of age-related macular degeneration (AMD), currently affects about 25,000 individuals in the United States [1]. SD is a primarily genetic recessive disorder that most typically has noticeable detrimental effects on central vision when the individual is between 10 to 20 years old [2]. Individuals with SD experience diminished central vision specifically in the macular region which is needed for sharp central vision. This commonly results in visual acuity between 20/100 and 20/400; thus, many people affected with this disease are legally blind [3]. Mied ziak and colleagues found that at the time of SD diagnosis, only about half of the sample felt they were adequately counseled [2]. Moreover, the majority of this sample reported depressive symptoms stemming from their vision loss.

Those with SD likely face stressors that are unique in comparison to those faced by individuals with other forms of blindness. For example, individuals with SD do not typically need a seeing-eye dog or a cane to navigate their surroundings, so it may appear that they can function in the same manner as a person with full vision, thus violating the stereotype of a visually disabled person. This stereotype violation may lead to increased stress in interacting with others because they may be perceived as a fraud, as is the case among those with AMD, a similar eye disease [4]. Individuals with more common forms of blindness, such as those with peripheral eye disease who demonstrate a need for navigational assistance [5], may be less likely to experience this stereotype violation.

Although the ability to navigate remains somewhat intact for those with SD, they may be unable to make simple recognitions of objects in their central line of vision. One consequence is an inability to identify faces [2], a disability that can be socially stressful. Individuals may fail to acknowledge a friend thus, causing the friend to feel slighted or instead, they may extend a warm personal greeting to a stranger they have falsely identified as a friend [6]. An indirect effect of having SD may lead to social embarrassment and cause the individual to question if they should disclose or conceal their disease to prevent social repercussions. Therefore, it is plausible that individuals with SD not only experience stress related to low-vision, but also experience stress associated with stereotypes, social embarrassment, and the fear of being misunderstood.

Minimal research has focused specifically on the psychological consequences of living with SD; this area is relatively unknown to both researchers and practitioners. The current study sought to gain insight on life with SD by analyzing essays produced in an expressive writing intervention. Over 200 studies have reported vast psychological and physical health benefits of EW in both community-based and chronically ill populations [7]; however, the written content of participant essays is typically disregarded as an intervention byproduct and remains relatively unexplored. The content of these essays could provide researchers with an untapped source of information related to the needs of the SD patients including the discovery of potential points of intervention and necessary health care improvement- pivotal information for both researchers and practitioners alike. The current study seeks to add to the literature related to the psychological and emotional issues related to progressive vision loss, by discussing the importance of the thematic content revealed among written essays from those with SD. A deeper understanding of the personal impact of vision loss specific to SD is warranted in order to investigate a more comprehensive disease management approach.

\section{Participants}

This study was approved by the Institutional Review Board at the University of Houston as part of a larger expressive writing (EW) study [8]. Participants were recruited between through multiple

Correspondence to: Jennifer L. Bryan Ph.D., 126 Heyne Building, University of Houston. Houston, TX 77204-5022, USA, E-mail: JenniferLynnBryan1@gmail.com

Received: January 21, 2016; Accepted: February 10, 2016; Published: February 13,2016 
avenues including online and in-person support groups (locally, nationwide, and internationally), and referrals from retina specialists registered with the American Society of Retina Specialists. A total of 22 participants completed written essays in which they wrote about their personal stressful experiences with SD without regard for spelling or grammar. Participants wrote in their own home at a time that was both convenient and conducive to private writing time. Participants ranged in age from 20-71 $(M=40.22, S D=13.42)$, and were primarily female (77.3\%), Caucasian (90.9\%), college educated (90.9\%), and residing in the United States (90.9\%). The remaining two participants (9.1\%) were from Denmark and the United Kingdom. Eligible participants were at least 18 years old, fluent in reading and writing English, and diagnosed with SD.

\section{Procedure}

The present sample was drawn from the experimental condition of an EW intervention study for individuals with SD. Eligible participants completed the informed consent process, and were enrolled in a study in which they were randomly assigned to one of two expressive conditions that differed only in writing instructions. Twenty-two participants in the experimental condition wrote about their most stressful SD related experiences for 20 minutes on three separate days.

Data from the 22 participants of interest was analyzed via a qualitative thematic content analysis, derived from principals of both Grounded Theory $[9,10]$ and Phenomenological Analysis [11]. Consistent with Grounded Theory, data was collected before creating hypotheses about the causes of stress for individuals with SD. Themes were generated by the data which revealed how participants perceived their stressful events, without strong initial interpretation from researchers. Layered analysis began with a group of five undergraduate research assistants (RAs) who independently coded all of the essays for main concepts. The RAs had no previous knowledge about SD to prevent bias. The RAs summarized the themes, and then two senior level graduate researchers compiled a list of themes the RAs noted. They then discussed and agreed that the list of themes fully represented the data and collapsed the themes when overlapping. The themes and original written essays were given to a second group of four RAs to individually verify the original themes and to select quotes that demonstrated the overarching themes. No additional themes emerged during the second round of analysis. All reported quotes are direct quotes from participants in the study and thus retain spelling and grammatical errors in order to maintain the integrity of participant responses.

\section{Results}

Below are the themes that were generated from the written essays about participants' experiences with SD along with quotes that give further insight to each of the themes described.

\section{Frustration with diagnosis process}

Eighteen participants reported experiencing negative emotions throughout the diagnosis process, from the initial doctor's appointments to coping with the SD diagnosis. Many participants recalled that eye doctors acknowledged something was wrong, but were unable to make a proper diagnosis. Or, if a proper diagnosis were made, questions and concerns were not addressed.

"I saw a doctor at least once a week every week. I was being told everything from I was going blind to I must have encountered a toxin that has damaged my eyes. Test after test was ran each seeming as inconclusive as the last." "was sent away with a list of retina docs in my state and area and a copy of a tesxtbook page descirbng Stargartds. I walked away confused and and disheartend"

Others reported having their psychological health questioned (or affirmed) when doctors were (un)able to identify SD. Many felt doctors failed to take their concerns seriously.

"the eye doctors I went to from around 10 years old to about 14 dismissed my claims and told my parents that I was making excuses for having not performing as well in school. After a couple of doctors suggested that I was either lying or exaggerating, my parents took me for a battery of psychological and intellectual tests."

"It has continued to irriate me that the first doctor I saw essentially blew me off and suggested it was psychological. If I had been a different sort of person, I might not have pursued the truth."

" $a$ weight lifted off my shoulders to be able to explain to everyone what I am going through"

In addition to struggles in the initial phases of the diagnosis process, many participants expressed intense negative emotions reminiscent of depression and anxiety following their final diagnosis. Several participants reported feeling helpless hopeless about their future in response to their diagnosis.

"I felt hopeless and depressed. I had already started having panic attacks and rather than my world expanding with boundless opportunity, it was shrinking by the day."

"I felt helpless, there was no information on what to expect - my doctor didn't know - wan not certain what I had except he thought it was a retinal disease."

\section{Loss of independence}

Fifteen participants identified their loss of independence as a frustrating consequence of SD. Losing the ability to drive was a commonly reported cause for their loss of self-sufficiency. For these individuals, it was more than just a transportation issue. Being unable to drive also prompted complicated emotional problems for many suffering from SD.

"I can feel my independence slipping away and I feel like it's eating away at me inside."

"By nature and upbringing I am an independent person. Not being able to find my way around felt awful, like I was losing a basic ability of living."

"I think one of the hardest parts about losing my vision, was giving up driving. It's such a part of your independence, it is not only a hassle to give up, it also hurts emotionally because you feel a loss of freedom."

As a consequence of being forced to depend on others, many individuals experience feeling burdensome to those who support them.

"The world is too big for a person who can't navigate it without a lot of help and I never have been good about asking for help. I hateabsolutely hate-feeling like I owe people yet that is how I am forced to feel because I am so dependent on others."

"Over the years, he has had to bear the burdon of doing all the driving, alwasy beeing the one to pick up a sick child or drive them to activities.It was a life changing event for both of us." 


\section{Social embarrassment}

Eleven respondents reported feeling ashamed or embarrassed of their disability. Some reported being unable to navigate the public world, often feeling as though others did not understand the challenges they experienced. Many reported public embarrassment when they made mistakes that involved other people such as mistaking a stranger for someone they knew because they were unable to properly perceive their face.

"The airport was a chaotic mess for anyone, but compound that by the inability to read the arrival and departure signs, it was a fiasco. ...I ended up asking EVERY kiosk in that wing if that gate was the one with my flight. The workers gave me weird looks like "come on you idiot, can't you read the sign." I felt flustered, stressed, humiliated, and overall aggrivated by the whole experience."

"Then there are the times that anyone with my condition can understand, which is walking up and giving someone a big hug or just a really over the top friendly greeting only to find out that it's a perfect stranger. Talk about embarrassing. Talk about feeling like an idiot. It's not like you even have the time to explain in those situations except to just say "So sorry, thought you were someone else".

\section{Feeling misunderstood}

Fifteen participants reported feeling that others were unable to understand the severity of their condition.

\section{"My wife just doesn't really get my disease."}

"I lost my sunglasses in the ocean once and $i$ was completely blind.. could not even open my eyes it was so bright and i know my boyfriend thought $i$ was exagerating or being dramatic"

Many participants felt additional stress when interacting with others because they did not appear as if they had an eye disease.

"I do have some vision. This at times makes it worse becasue there is a certain expectation that doesn't exist with people that are completely blind."

"best part was my co workers just sat around saying how $i$ was "faking" my eye disease and that im crazy etc etc. why and how would $i$ make up this diagosis. why would $i$ want this?"

\section{Effects on personal and family life}

Twenty-one participants reported concerns about how SD affected those close to them. Others reported difficulty forming close relationships because they felt overlooked due to their disability. Those who were able to form romantic relationships had a hard time dealing with the imbalance of responsibility on the partner who is free of the disability. Some spouses reported feeling inadequate and unable to sufficiently support their spouses in household and parental responsibilities because of their SD.

"One thing I have found in my 17 years of being legally blind, relationships can be more challenging. The beginning of the end of my marriage was my vision loss. It has been very difficult to find someone who does not seem to mind that my vision is far from perfect. No one wants to take the time to get to know me, all they see is a disability. I feel that I have to work harder to show people who I am. I am more than a blind girl."

"I believe that my wife has been short changed because she is married to a blind man: a man who cannot drive, fixe things, and is a poor lover.
I foten think about my inability to do simple things with my kids like play catch or take out on a trip. It is even hard for me to tell if there is something wrong with them becasue I can't recognize certain signs"

"Who in their low 20's envisions their spouse being legally blind in 10 or 20 years? I felt like damaged goods."

Another individual reported feeling taken advantage of by her romantic partner.

"He had been having an affair for 9 months. I was devastated! But what made it even worse is that he took advantage of my lack of site."

Concerns about responsibilities as a spouse and parent were not the only source of concern for those with SD. Some worried about the genetic component fearing they would pass the disease onto their children. Some even worried that a relationship partner would not want them because of this risk. Those who already had children when they were diagnosed with SD had similar fears that their children may already have the disease, and felt guilty.

"If this was genetic, would he wantt to spend his life with a woman who could possible pass this to his children?"

"On top of all this I worry more and more about the herefitary aspect of my condition. How will I feel if I have passed Stargardt disease on to her? How will she feel about that too? Will she resent me because of it? "

Young individuals with SD hoping to have a family feared how their disease may affect their ability to parent. Some even worried they may be unable to save their child in a dangerous situation.

"As a newly marriend woman my husband and I are preparing to have children. I wonder how it will be for children living with amom who is legally blind. Will I be a good mother without full sight? What if my child is choking or has a rash, will I be able to tell and protect him or her?"

\section{Effects on professional life}

The professional world is another realm in which individuals with $\mathrm{SD}$ often struggle, as they endure both direct and indirect consequences of their disability. Participants reported that SD negatively impacted their professional life, including the inability to obtain and maintain employment. Those who were able to maintain employment reported mistakes because of their low vision.

"I lost my job as a nurse because $i$ told my manager $i$ was having problems reading the computer and charting. they didn teven try to help me out or accomadate me"

"A while after that, I got a job... as a receptionis at an optomestrist's office. I told them I had an eye disease -- wrote it at the top of my job application. They knew. Anway, at the end of the first week, they fired me, saying I was looking too closely at the apperwork, and that it looked bad for them as optometrista, as if they couldn't make glasses for everyone."

Employees and students suffered the consequences of their disease even though the progression and manifestation of the disease is out of their control. Many experienced anxiety about the future and what future difficulties their eye disease might cause. Others suffered from discrimination from peers. Thus it is understandable that some individuals develop social anxiety regarding relationships in the workplace, fearing that others would learn of their disability and judge them. 
"I am a college student right now, undergrad, but i plan on going to grad school and then working to get my Ph.D. after that. $i$ have had many sturggles with trying to find a job because of my vision limitations, and that made me worry a lot about my career because what would $i$ do if $i$ worked so hard and fought through so many struggles, and then when $i$ finally got to the point where $i$ was doing what $i$ absolutely loved, $i$ couldn't do some of the things because of my low vision. that would be heartbreaking"

"The worst part of Stargardt disease for me is dealing with work. I am afraid to tell anyone at work about my condition"

"It's embarrassing at work as I mistake colleagues for other people, or say hello to the same person numerous times not knowing who they are. I don't want the entire workplace knowing about mu disability, but nor do I want people to think that I am stupid or ignorant."

\section{Coping and social support}

Fifteen respondents named several different strategies for coping with SD including working through acceptance, finding humor in challenging situations, and seeking out social support in the form of close relationship partners and support groups.

"Despite the fears and concerns I have had in the past, I am at a point in my life where I have accepted this disease and and have overcome many of the obstacles associated with it. I use humor and honesty to continue through my life and cope with having a disability. This has made it much easier for me to be successful and advocate for myself."

"I began to look for adavaccy and support groupd. I moved through the grieving process of my eeysight quickly by nature and choice. I needed to grab some control over my life and not let this diagnosis control me!"

"But people are kind; many are helpful. And riding the buses I see a whole lot of people who struggle more than"

"It isn't a situation I would choose, but it is one that I've been given and I'm going to deal with it the best I can. It helps to stay positive and connect with other people dealing with the sane struggles and obstacles that I deal with on a daily basis."

"I was fearful of how my boyfrined my see the situatoin. Would this man who I so badly wanted a future with reject the idea now? ... Fortunately, he felf the opposite and actually proposed to me shortly after [I was diagnosed] stating "I could have cancer tomorrow and I know you wouldn't thingk twice and that's the kind of woman I want to be with."... Knowing that I had his love and support allowed me to ope up more to my frineds and to find a new love and acceptacne for my life as it would be."

\section{Finding benefit in the SD experience}

Thirteen respondents were able to identify ways in which SD had a positive influence on their life. One common response was regarding memory; many participants reported developing a better memory than those around them to make up for their inability to see. Others reported having to overcompensate in other sensory areas to make up for their poor eyesight.

"I have always had a very good memory, but over the last decade, if I focus on reading something, I retain it to long term memory pretty much everytime on the first read. Also my verbal communication skills are great. I not only retain what others are syaing very weill, I have learned to read emotions, intent, everything in how people say things, what words they say, and Iknow that is due to the fact I can't read their

\section{facial expressions."}

Some participants mentioned their relationships had been improved by their disability.

"I think losing my vision has also helped me to see people better. I am able to see more with my heart and I think that I am able to see the true person and not the person they want people to physically see."

"I have two beautiful successful children in their twenties and they claim they are more independent because they had a mom who couldn't always do what the other mom did. (drive, read to them, cook with them)."

"I feel my husband and I are closer than other couples due to the fact that whenever the kids had to go somewhere, we couldn't split up and take the kids to different events we all had to go together."

\section{Discussion}

The current study provides preliminary insight into the lives of individuals with SD, a rare eye disease, by utilizing essays produced in an expressive writing intervention. As part of this intervention, participants revealed their deepest thoughts and feelings, about their most stressful experiences related to SD. Although participants reported a wide array of experiences, many similarities arose during content analysis. The most commonly expressed stressful experiences included frustration with the diagnosis process, loss of independence, frequent social embarrassment, feeling misunderstood, and negative effects on their personal and professional lives.

Participants reported feeling stress in multiple domains starting at the diagnosis process. Consistent with previous research that only about half of individuals with SD are diagnosed within one year of seeking medical attention [2], many participants had difficulty being diagnosed. Even though low-vision symptoms are typically apparent to the individual (i.e., blurred vision, having to sit close to the TV or reading materials), many patients were given an incorrect diagnosis, no diagnosis at all, or were told it was psychological, discounting their physical vision loss altogether. Once diagnosed, many participants walked away from their eye care specialist without a clear understanding of what SD actually is and how to physically, emotionally, and mentally cope. Practitioners may improve their care by educating their patients on the disease, or following up the diagnosis appointment with a phone call to answer questions the patient may have after having time to process their diagnosis. In addition, once participants received the diagnosis, many reported their independence slipped away (e.g., losing their driver's license). This loss often prompted severe emotional distress. Understanding the emotions that come with a $\mathrm{SD}$ diagnosis can equip care providers of all kinds (family members, friends, physicians) to help the patient; patients may also benefit from psychological service referrals to seek help in handling the emotional distress related to diagnosis.

Interacting with others was also reported as a frequent cause of SDrelated stress; participants felt embarrassed and misunderstood when it came to their eye disease. Respondents listed a myriad of potentially embarrassing social situations that occur daily from misidentifying a stranger and greeting them like a close friend, to being unable to read signs and needing assistance to navigate unfamiliar areas. Practitioners may wish to refer individuals diagnosed with SD to a vision rehabilitation therapist, who teaches skillsets that can improve daily functioning and provides access to supportive technology that empower individuals to live safe, meaningful, and autonomous lives. 
No participant wrote about being referred and this population may greatly benefit from these services. In addition to vision care providers, participants reported how social support helped to alleviate their stress. These findings are consistent with previous research showing that those with higher social support have better adjustment to living with low-vision [12], psychological functioning [13], life satisfaction, and fewer depressive symptoms [14] than those with low support. Likewise, our study revealed that the support of loved ones, or lack thereof, had a major impact on those diagnosed with SD. Some reported their diagnosis brought their family closer and revealed true friends, while others reported the diagnosis tore their family apart. To counteract the interpersonal stress and feelings of being misunderstood in relation to diagnosis, practitioners should consider referring their patients to SD support groups so that these patients can talk to others who have a personal understanding of their unique experience.

Participants expressed fears about how the disease would affect their personal and professional lives. These fears included questions such as whether to tell their boss about their disability and wondering how their low-vision would affect what careers they could pursue. Common fears arose about being fired from the careers they love and being ridiculed about catastrophizing the severity of their disease. Perhaps more importantly, in the personal realm, many feared they would be unable to be a good spouse or would pass SD on to their children. A decision that many people faced was whether to disclose their diagnosis to others including friends, family, and even acquaintances. Despite all of these negatives, participants also expressed positive experiences related to SD including feeling closer to their families and finding unknown strength within themselves. Researchers can greatly improve the lives of those with SD by developing interventions to target their fears; these interventions could emphasize developing effective communication and coping strategies to address interpersonal conflicts that stem from their vision loss in all aspects of life.

Although our current findings suggest that those with SD may experience some of the same challenges as those with AMD, they also face unique challenges specific to their younger age group including personal concerns related to relationships and starting a family, and professional concerns surrounding their budding career goals. This demonstrates a need for research on the psychological aspects of living with SD above and beyond what is already offered in the AMD literature.

This study provides a window into the emotional world of individuals with SD- a domain that many individuals choose to conceal. These findings have greater implications for intervention research and practitioners. AS SD is a rare disease, many may never meet another person with their condition. In the future, researchers should investigate the psychological and physical health benefits stemming from interventions involving those with SD sharing their experiences with one another (e.g., in online forums). Interventions like these may reduce the stress associated with feeling misunderstood.

These findings also have implications for practitioners. A large portion of our sample reported frustration with their eye care specialists' lack of concern for their psychological well-being. Many individuals with SD experience psychological distress throughout the process of diagnosis, and adjustment thereafter. Some participants reported feeling burdensome to their loved ones because of their vision disability. This finding is particularly concerning considering feeling burdensome to others has been related to negative physical and psychological health outcomes including pain [15], depression, suicide ideation and suicide-related behaviors [16]. Eye care practitioners could go beyond the physical aspects of SD (i.e., making sure the individual understands SD), but also introducing other services that will focus on the psychological consequences of living with SD (e.g., vision rehabilitation specialist). Attending to patients' psychological needs may be especially important for eyesight rehabilitation considering recent findings suggest even minimal depressive symptoms may be related to decreased visual function [17]. The themes expressed in these writings can inform vision rehabilitation programs and retina specialists about potential stressors those with SD face, better preparing them to address the concerns of newly diagnosed patients and families. Although SD is a specific form of vision loss, understanding "the SD experience" may allow researchers to understand the experiences of individuals with other forms of legal blindness or chronic conditions that are easily concealed from others.

Moreover, fully utilizing an EW intervention has several benefits for individuals diagnosed with rare diseases. Through the process of writing, those with SD are able to experience a range of positive health benefits [8]. After the intervention is complete, researchers and health care providers are privy to the authentic experiences of the individual through that expressed during the writing sessions. This information may shed light on potential gaps in care.

The strengths of this research should be considered in light of its limitations. SD is a rare eye disease, making it difficult to recruit a large number of eligible participants. We made significant efforts to recruit participants through multiple venues in order to obtain a representative sample. Although we did not provide monetary compensation for this study, participants seemed very enthusiastic, indicating that individuals with SD may feel the need to share their experiences. Furthermore, we allowed participants to write whatever came to mind and whatever they wanted to share. It is possible that participants would have reported a larger amount of similar experiences if we had asked them to answer specific questions during the writing sessions. Collecting the data online provided participants the privacy to express themselves fully without fear of judgment; however, participants may have disclosed more if they were speaking to a confidential interviewer. Future research may employ structured interview methods to answer more specific research questions.

\section{References}

1. National Eye Institute. More Americans Facing Blindness Than Ever Before Report Released On One of the Most-Feared Disabilities. Retrieved from http://www.nei.nih. gov/news/pressreleases/032002.asp 2002.

2. Miedziak AI, Perski T, Andrews PP, Donoso LA (2000) Stargardt's macular dystrophy-a patient's perspective. Optometry 71: 165-176. [Crossref]

3. Health., N.I.o., Stargardt Mmacular Degeneration, Retrieved from http://ghr.nlm.nih gov/condition/stargardt-macular-degeneration. 2009.

4. Elaine YHW, Robyn HG, Jennifer BH, Jill EK (2004) The Experience of Age-Related Macular Degeneration. J Vis Impair Blind 98: 629-640.

5. Szlyk JP, Fishman GA, Grover S, Revelins BI, Derlacki DJ (1998) Difficulty in performing everyday activities in patients with juvenile macular dystrophies: comparison with patients with retinitis pigmentosa. Br J Ophthalmol 82: 1372-1376. [Crossref]

6. Cynthia O, Gerald M, Kay S, Laura ED, Carolyn B (2006) Focus groups with persons who have age-related macular degeneration: Emotional issues. Rehabilitation Psychology 51: 23.

7. Frattaroli J (2006) Experimental disclosure and its moderators: a meta-analysis Psychol Bull 132: 823-865. [Crossref]

8. Bryan JL, Lu Q (2014) Vision for improvement: Expressive writing as an intervention for people with Stargardt's disease, a rare eye disease. J Health Psychol. [Crossref] 
9. Glaser BG, Strauss AL (1967) The discovery of grouded theory. Chicago (US): Aldine.

10. Strauss AL, Corbin JM (1998) Basics of qualitative research: techniques and procedures for developing grounded theory. Basics Of Qualitative Research: Techniques And Procedures For Developing Grounded Theory.

11. Giorgi A (1985) Phenomenology and psychological research. Duquesne University Press.

12. Davis CJ, Lovie-Kitchin, Thompson B (1995) Psychosocial adjustment to age-related macular degeneration. $J$ Vis Impair Blind 89: 16-27.

13. McIlvane JM, Reinhardt JP (2001) Interactive effect of support from family and friends in visually impaired elders. J Gerontol B Psychol Sci Soc Sci 56: P374-P382. [Crossref]
14. Reinhardt JP (1996) The importance of friendship and family support in adaptation to chronic vision impairment. J Gerontol B Psychol Sci Soc Sci 51: P268-278. [Crossref]

15. Kowal J, Wilson KG, McWilliams LA, Péloquin K, Duong D (2012) Self-perceived burden in chronic pain: relevance, prevalence, and predictors. Pain 153: 1735-1741. [Crossref]

16. Joiner T (2005) Why people die by suicide. Harvard University Press.

17. Rovner BW, Casten RJ, Hegel MT, Leiby BE, Tasman WS (2007) Preventing depression in age-related macular degeneration. Arch Gen Psychiatry 64: 886-892. [Crossref]

Copyright: (C2016 Bryan JL. This is an open-access article distributed under the terms of the Creative Commons Attribution License, which permits unrestricted use, distribution, and reproduction in any medium, provided the original author and source are credited. 